\title{
Tuberculosis multidrogoresistente
}

\section{Tuberculosis}

Rosa Pinargote Chancay. MG. EPI ${ }^{(1)}$

Yasmin Castillo Merino MG.G.S ${ }^{(2)}$

Virginia Pincay Pin MG.G.S ${ }^{(3)}$

Adriana Castillo Merino MG.G.E ${ }^{(4)}$

(1) Universidad Estatal del Sur de Manabí, Ecuador

(2) Universidad Estatal del Sur de Manabí, Ecuador

(3) Universidad Estatal del Sur de Manabí, Ecuador

(4) Universidad Estatal del Sur de Manabí, Ecuador

Contacto: hugoandresyepez@ hotmail.es,yascasme@hotmail.es

Receptado: 13/07/2014 Aceptado: 012/09/2014

\section{Resumen}

La tuberculosis es una enfermedad de transcendencia social que tiene una importante morbilidad y mortalidad en el mundo entero, su manejo ha llegado a ser más complejo debido al incremento de resistencia a las drogas antituberculosas comúnmente usadas para su tratamiento como la isoniazida y rifampicina que son las más poderosas para el tratamiento de esta enfermedad. Se han establecido cuatro categorías diferentes de farmacorresistencia: Monorresistencia.- que es la resistencia a un fármaco antituberculoso. Polirresistencia: resistencia a más de un fármaco antituberculoso diferentes de la isoniazida y la rifampicina. Multidrogorresistencia: resistencia a la isoniazida, rifampicina o extendida a algunas fluoroquinolona y por lo menos uno de los tres fármacos inyectables de segunda línea (capreomicina, kanamicina y amikacina). La epidemiología de la TB-MDR es el análisis de la distribución temporo espacial y los factores que determinan la presencia de nuevos casos en nuestras poblaciones y adoptar estrategias de prevención ante la diseminación de este tipo de TB.

Palabras clave: Tuberculosis resistente a múltiples medicamentos

\section{Tuberculosis multidrug}




\begin{abstract}
Tuberculosis is a disease of social significance that has significant morbidity and mortality worldwide, its management has become more complex duce to increased resistance to antituberculosis drugs commonly used for treatment such as isoniazid and rifampicin are the most powerful in the treatment of this Sexually transmitted diseases. We have established four categories of drug resistance: Monorresistencia.- which is the resistance to antituberculosis drug. Poly resistance: resistance to more than one different antituberculosis drug isoniazid and rifampicin. Multidrug resistance: resistance to isoniazid, rifampicin or extended to some fluoroquinolone and at least one of three injectable second-line drugs (capreomycin, kanamycin and amikacin). The epidemiology of TB-MDR is the analysis of the distribution temporal and spatial factors that determinate the presence of new cases in our populations and adopt prevention strategies to disseminate this type of TB.
\end{abstract}

Keywords: multi-drug resistant tuberculosis.

\title{
Introducción
}

Según el Ministerio de Salud y la Organización Mundial de la Salud (OMS) Se considera que cada persona que enferma de TB sin tratamiento efectivo, contagia el bacilo tuberculoso entre 10 a 15 personas cada año, y que las cepas resistentes y extremadamente resistentes se transmiten de la misma manera que las cepas sensibles.

Desde 1990, el número de casos de tuberculosis notificados en Ecuador ha ido en aumento, pero con tendencia irregular, caracterizada por rápidos incrementos y descensos. (salud, 2011) Para el año 2000 la TB constituyó la décima causa de morbilidad y muerte entre los hombres y mujeres. En ese año, la tasa de mortalidad por TB fue 0.089 por 100,000 habitantes (salud, 2011)

\section{Objetivo general}

Exponer las causas que influyen en la presencia de la TB - MDR en la Provincia de Manabí

\section{Objetivos específicos}

- $\quad$ Conocer el número de casos de TB MDR en la Provincia de Manabí

- Identificar los factores que determinan la presencia de este tipo de TB 
- Analizar los mecanismos de resistencia a los medicamentos del bacilo TB

\section{Desarrollo}

La resistencia antimicrobiana es un fenómeno común a todos microorganismos, formando parte de su respuesta frente a la agresión externa; en este caso, la tuberculosis (TB) resistente es una respuesta defensiva del M. tuberculoso frente a los antimicrobianos.

El tratamiento para el M. tuberculoso requiere de un esquema combinado de tres o cuatro drogas, por lo que la resistencia aislada a una de ellas no necesariamente va a generar un fracaso terapéutico, sin embargo hay que tomar en cuenta que no todos los medicamentos antituberculosos son igualmente eficaces y si podrían llevar al fracaso del tratamiento.

Se define como Tuberculosis multidrogo resistente (TB MDR) cuando existe resistencia a isoniacida (INH) y rifampicina (RFP).

Estas formas de resistencia, se encuentran muy extendidas en el mundo, dificultando el control de la TB en los últimos 20 años a nivel mundial. La TB es considerada una enfermedad reemergente y un problema de salud pública mayor. A pesar del esfuerzo de la comunidad internacional en la mayor asignación de fondos, el desarrollo de nuevos métodos diagnósticos, y la investigación en nuevas drogas, el panorama para el control de la TB no se ve claro a mediano plazo.

La resistencia a los medicamentos anti-TB es de tipo cromosómica, y fue descubierta tempranamente en los años 50s, siendo observada primariamente frente a la STM. Luego se apreció que la combinación de fármacos, hacia posible la eficacia terapéutica de los esquemas de medicamentos, surgiendo así la 1ra base bacteriológica de la terapéutica en TB "combinación de fármacos", que evitaba la selección de cepas resistentes. Así mismo, se apreció una mayor frecuencia de recaídas ante la descontinuación temprana de los esquemas, por lo que la "extensión de la duración del tratamiento" es considerada la segunda base terapéutica de la TB, la cual podía llegar a extenderse a 12 meses o más.

La aparición de la RFP, en asociación a los otros fármacos (en especial a la INH) logra un esquema potente, por lo que el esquema se reduce a 6 meses, dando paso al llamado Esquema 1 de la Organización Mundial de la Salud (OMS), sobre el cual descansa la Estrategia DOTS 
(Directly Observed Treatment Short-curso. Es importante recordar que la estrategia DOTS considera para su buena aplicación, un compromiso político de los gobernantes para garantizar la disponibilidad de las pruebas de diagnóstico y medicamentos, así como la aplicación del tratamiento de manera supervisada, todo ello para garantizar un tratamiento eficiente y evitar la resistencia. (V., 2010) Por ello se buscó expandir la Estrategia DOTS a nivel mundial. Sin embargo, a pesar de los esfuerzos, la TB MDR surge en todos los continentes y se expande, aun en los lugares donde la estrategia DOTS se aplicaba con eficiencia; por lo que la OMS ve la necesidad de propiciar el tratamiento con drogas de 2da línea para pacientes en esta condición.

Así mismo, debido al elevado costo del tratamiento, se generan mecanismos que facilitan el acceso a drogas de segunda línea, bajo supervisión y control el cual está a cargo del Comité de Luz Verde, sin embargo este esfuerzo, también tiene sus limitaciones y aparece la TB XDR. Es por ello, que se considera insuficiente la sola aplicación de la estrategia DOTS para el control de la TB en el Mundo, y se comienzan a adicionar nuevas estrategias. Sin embargo, la mayor parte de ellas siguen considerando un fuerte enfoque biomédico, que hasta el momento no ha mostrado eficacia en los países pobres del mundo. (V., 2010)

La resistencia un problema complejo en la prevención y control Además de las características biológicas del germen y los aspectos epidemiológicos relacionados a su trasmisión, existen factores biológicos en el propio huésped que perturban la eficacia de los antimicrobianos y por lo tanto favorecen la resistencia.

Los grupos de mayor riesgo de TB MDR se consideran los siguientes: a) Antecedente de ser contacto de paciente con TB MDR confirmada con Prueba de Sensibilidad (PS) o en tratamiento con drogas de segunda línea, b) Inmunosupresión asociada a exposición intrahospitalaria: Infección VIH, Diabetes Mellitus, insuficiencia renal crónica, neoplasias, enfermedades terminales, tratamiento crónico con corticoides, otras; c) Recaída en menos de seis meses de egresar como "curado" del Esquema Uno de tratamiento; d) Paciente con tuberculosis multitratada (dos o más tratamientos); e) Personal de salud que incluye estudiantes de ciencias de la salud y promotores de salud que trabajan en TB; f) Residir en los últimos dos años en una zona de elevada prevalencia de TB MDR, g) Población privada de libertad o con antecedentes del mismo; h) Trabajador de establecimientos penitenciarios; i) Usuarios de drogas ilegales; j) Contacto de paciente fallecido por tuberculosis antes tratada; k) Pacientes con antecedente de tratamiento previo particular y/o auto administrado mayor de 30 días; 1) Paciente con antecedente de irregularidad al tratamiento; m) Antecedente de hospitalización previa en salas 
Rev. SINAPSIS, Vol. 5, No 2, Diciembre 2014

ISSN $1390-7832$

de medicina o emergencia, por más de una semana, por lo menos una vez en los últimos dos años; n)Tratamiento previo con presencia de Reacción Adversa a Fármacos Antituberculosos (RAFA) que obligó a cambiar a dosis sub óptimas y/o suprimir algún medicamento; o) Contacto de paciente que fracasó a tratamiento antiTB .

\section{Características clínicas}

Originalmente las características clínicas de un paciente con TB MDR, mostraban a un paciente caquéctico, en mal estado general, con insuficiencia respiratoria crónica, que en algunos casos se asociaban a signos de Cor pulmonale, donde la radiografía de tórax evidenciaba compromiso pulmonar extenso de aspecto fibrocavitario, usualmente bilateral. Este paciente era producto de uno o varios tratamientos generalmente asociado a abandono en varias oportunidades, todo ello facilitando la generación de resistencia de sus cepas (resistencia adquirida). (Ignacio, 2010)

Sin embargo, en el seguimiento de un paciente que inicia tratamiento TB, en quien ignoramos su perfil de sensibilidad, apreciamos algunas características clínicas que nos pudieran sugerir una mala evolución, y por lo tanto la presencia de TB MDR (siempre y cuando el paciente haya sido adherente): no ganancia de peso, persistencia de tos, falta de negativización de la baciloscopía al final del segundo mes de tratamiento, volver a hacerse positiva la baciloscopía, especialmente ante la persistencia de cavidades o lesión pulmonar evolutiva. (Ignacio, 2010)

\section{Diagnóstico.}

El diagnóstico de la TB resistente, se realiza únicamente a través del resultado de una prueba de sensibilidad antimicrobiana, que indica resistencia a una o más drogas.

En los países desarrollados, en todos los casos, sea cual fuere la forma clínica de tuberculosis, se le realiza cultivo y prueba de sensibilidad. En los países en desarrollo (debido a los escases de recursos económicos y capacidades de laboratorio), a los casos nuevos se les prescribe un tratamiento estandarizado de primera línea "Esquema 1"

Sin embargo, la determinación de la sensibilidad antimicrobiana, es compleja, debido a que el M. tuberculosis es un germen de reproducción lenta. Por lo que se requiere de 4 a 6 semanas para su crecimiento en cultivo, y semejante tiempo para la determinación de la sensibilidad por el método convencional "método de proporciones".

Así, se buscaron pruebas más económicas y fáciles de desarrollar en países en desarrollo, obteniéndose las pruebas rápidas MODS y GRIES, las cuales son pruebas fenotípicas, es decir 
Rev. SINAPSIS, Vol. 5, No 2, Diciembre 2014

ISSN $1390-7832$

se observa directamente in vitro el fenómeno de la resistencia de las cepas evaluadas. Estos métodos permiten determinar la sensibilidad (o resistencia) de INH y RFP, es decir nos dicen si la cepa "es o no es TB MDR" en pocas semanas (1 a 4 semanas). Estos métodos, además de su rapidez, son económicos y por lo tanto muy útiles.

Por otro lado, la biología molecular permite identificar genes específicos de resistencia en el M. tuberculosis para algunos medicamentos, lo que trae consigo, la disponibilidad de pruebas genotípicas para la determinación de la resistencia. Así, se encuentra que para la RFP el gen rpo B está asociado a > 95\% de las cepas resistentes y para la INH los genes kat G, inh A en el 85\% de dichas cepas. Estas determinaciones se pueden hacer en 3-6 horas (GenoType MTBDRPlus-Hain lifescience, GERMANY), o en 2 horas (Cepheid Xpert MTB/RIF), siendo su mayor inconveniente el costo.

Estas pruebas rápidas, tanto fenotípicas, como genotípicas, en el momento actual, solo nos permiten tener información sobre INH o RFP, por lo que en áreas poblacionales donde existen cepas TB XDR o cepas pre-XDR (cepas TB MDR con resistencia adicional a algún inyectable o quinolonas) su ayuda se torna limitada (no nos permite determinar la resistencia a los otros antimicrobianos), teniendo que decidir un tratamiento, que no podremos modificarlo sino hasta 12 semanas después (cuando dispongamos de la pruebas de sensibilidad por el método de proporciones). De cualquier manera, estas pruebas rápidas, cuando expresan sensibilidad a INH y RFP, definen ausencia de TB MDR, y por lo tanto el esquema de primera línea a recibir será seguro. (Muyason Oblitas, 2009)

La prueba de biología molecular disponible en el INS, es GenoType MTBDRPlus ®-Hain lifescience, GERMANY. El costo de la prueba y las capacidades actuales, aún no permiten su amplio uso a nivel nacional. Actualmente los centros de salud, la solicitan para todos los casos nuevos de TB, restringiéndose para los otros centros en caso exista sospecha de TB MDR. Así mismo, estas pruebas ayudan en pacientes que han iniciado tratamiento sin un cultivo previo, o cuando hay sospecha de fracaso (resistencia) encontrándose bajo tratamiento (que pudiera impedir el aislamiento de la cepa por cultivo).

Si bien, se está disponiendo cada vez de mejores capacidades para el diagnóstico de la resistencia en TB, se van complejizado cada vez más los procesos en el nivel operativo. Así, en los casos nuevos de TB iniciamos con un esquema 1 y luego con los resultados de las pruebas 
rápidas que muestran TB MDR (MODS, GRIES o GenoType) lo cambiamos 2 ó 4 semanas después a un esquema estandarizado TB MDR, y es posible que 10 a 16 semanas después debamos de modificar el esquema con el resultado del método de proporciones, que nos precisa claramente el perfil de sensibilidad a drogas de primera y segunda línea. De esta manera, se genera un sistema de envío de muestras a los laboratorios regionales y/o INS, procesamiento de muestras en dichos laboratorios, conocimiento del resultado por el médico tratante, decisión del nuevo esquema, disponibilidad del esquema en el centro de salud correspondiente, y finalmente la toma del tratamiento por el paciente; todos estos procesos con riesgos de retrasos y errores. Además, la decisión del esquema pasa por la evaluación Comité de Evaluación de Retratamiento Intermedio (CERI) y simultáneamente del Comité de Evaluación de Retratamiento Nacional (CERN), que si bien busca una decisión terapéutica adecuada, enlentece el inicio del tratamiento del paciente de manera importante. Actualmente, la nueva gestión en la Estrategia Nacional de Prevención y Control de TB ha buscado disminuir el retraso, facilitando que los CERI decidan las posologías, disponiendo el inmediato envío de las medicinas al centro de salud.

La decisión del esquema de tratamiento para TB resistente, debe de hacerse en todos los casos con una prueba de sensibilidad para drogas de 1ra y segunda línea, las cuales orientan la selección de drogas. En general los medicamentos de segunda línea, son menos potentes, más tóxicos y más caros que las medicaciones para TB sensible; requiriendo un uso prolongado para alcanzar la curación, recomendándose al menos 18 meses de tratamiento.

Retratamiento individualizado para TB MDR. (Muyason Oblitas, 2009)

El esquema se basa en los resultados de la prueba de sensibilidad del paciente, para fármacos de primera y segunda línea. En la elaboración de estos esquemas se considera una fluoroquinolona (Moxifloxacino, Levofloxacino) + un Inyectable (Kanamicina, Capreomicina, Amikacina, Estreptomicina) + 1 droga de segunda línea oral (Cicloserina, Etionamida, PAS), + drogas de primera línea: Ethambutol, Pirazinamida. Se puede agregar 2 ó más de las drogas del Grupo 5 (Tabla 2) según el patrón de resistencia si no se logra alcanzar un esquema con por lo menos 3 drogas efectivas (por lo menos dos de ellas bactericidas). La duración del tratamiento es de 18-24 meses, pudiendo aún extenderse por más tiempo. 
Rev. SINAPSIS, Vol. 5, No 2, Diciembre 2014

ISSN $1390-7832$

Este esquema se indica sin disponer de los resultados de la PS del paciente, cuando se encuentra en mala condición clínica, y tenga al menos un cultivo positivo o prueba de GenoType. Las indicaciones son en: i) Paciente con TB (nuevo o antes tratado) contacto intradomiciliario de caso índice TB MDR, ii) Paciente con TB en esquema 1, que tiene un resultado de MODS, GRIESS o GenoType que indica resistencia a $\mathrm{R}$ y $\mathrm{H}$.

Para la elaboración de dicho esquema se consideran: a) El antecedente de fármacos previamente recibidos, b) La prueba de sensibilidad del caso índice TB MDR documentado, c) El esquema de retratamiento recibido por el caso índice y su condición de egreso, si lo hubiera, d) El patrón de resistencia local (áreas de alto riesgo) o regional.

El esquema debe ser modificado al recibirse el resultado de la PS del método de proporciones, pasándose así a un esquema individualizado. Si el resultado de la PS muestra pansensibilidad debe pasar al esquema 1. Si no se logra disponer de la PS, el tratamiento puede extenderse a 24 meses o más.

\section{Retratamiento estandarizado para TB MDR}

El esquema comprende: 2- 4 Km Lfx Z E Eto Cs/ Km2-3 Lfx Z E Eto Cs/Lfx Z E Eto Cs [2 a 4 meses de Kanamicina + Levofloxacina + Pirazinamida + Ethambutol + Etionamida + Cicloserina (todos diario); seguido de Kanamicina 2 a 3 veces por semana + Levofloxacina + Pirazinamida + Ethambutol + Etionamida + Cicloserina (todos diario); seguido de Levofloxacina + Pirazinamida + Ethambutol + Etionamida + Cicloserina (todos diario)]

Está indicado en pacientes con sospecha de TB MDR, y cuya PS está en proceso; en los siguientes casos: i) Paciente que fracasó al Esquema 1, demostrado por cultivo positivo al $4^{\circ}$ mes de tratamiento; ii) Paciente con TB activa y antecedente de dos tratamientos previos completos y que cuenta con cultivo positivo; ó en iii) Paciente en Esquema 1 en muy mal estado clínico y con sospecha de TB MDR.

El esquema debe ser modificado al recibirse el resultado de la PS, pasándose así a un esquema individualizado. Si el resultado de la PS muestra pansensibidad debe ser pasado a esquema 1. Si no se logra disponer de la PS, el tratamiento puede extenderse a 24 meses o más. Se requiere una vigilancia epidemiológica que incida en los aspectos sociales, que permitan generar nuevas estrategias de intervención considerando "los determinantes sociales". 
Rev. SINAPSIS, Vol. 5, No 2, Diciembre 2014

Un enfoque intenso sobre poblaciones vulnerables en riesgo de $\mathrm{TB}$, que a la vez constituyen el mayor riesgo de abandono de sus tratamientos TB (Personas sin hogar, usuarios de drogas, alcohólicos, personas que recientemente recuperan su libertad, etc), necesita ser implementado.

\section{RESULTADOS}

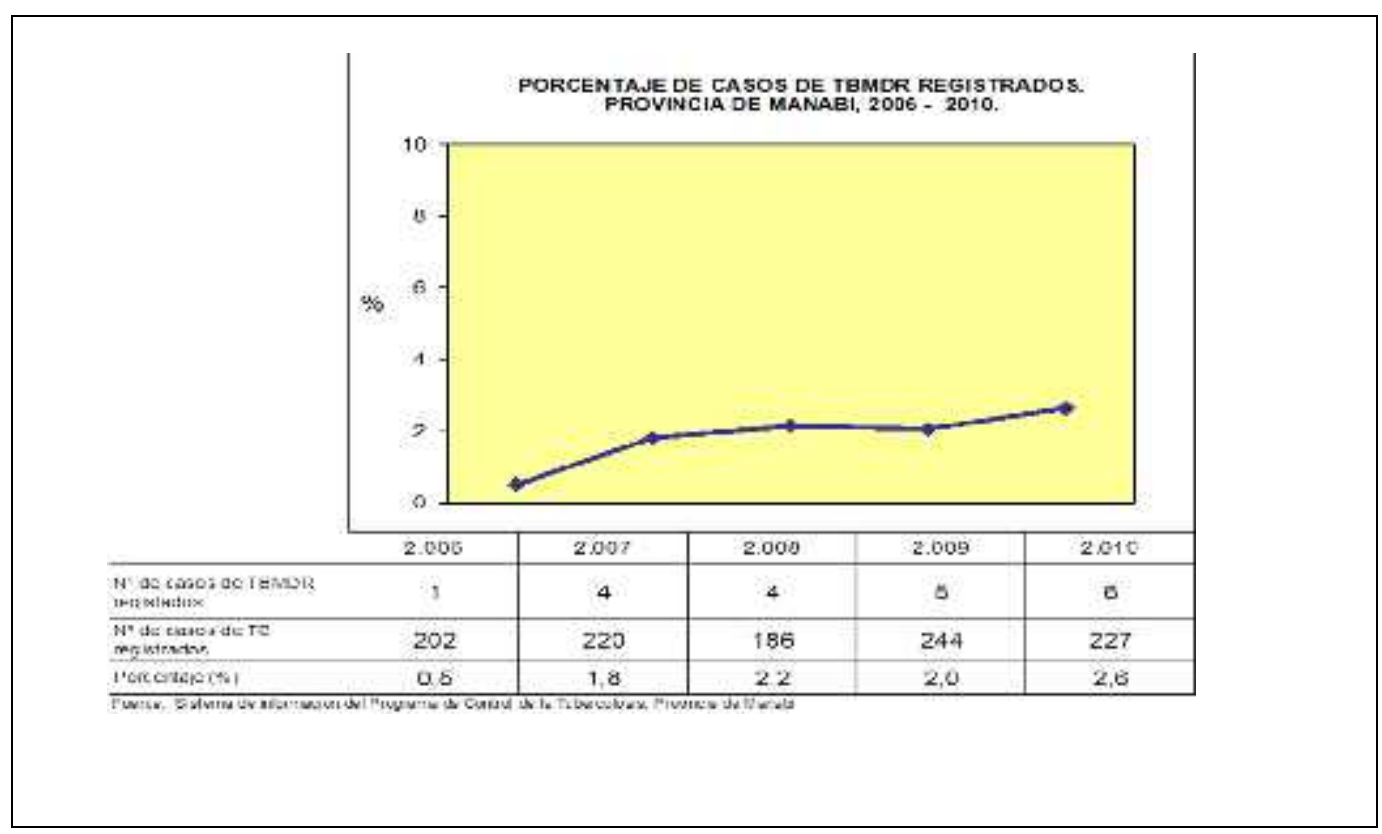

De los 1097 casos de Tuberculosis registrado desde el 2006 hasta el 2010 el 1,82\% de los casos corresponden a pacientes con Tuberculosis Multidrogoresistente.

\section{Discusión}

Una de las causas para la resistencia primaria o inicial se da en pacientes que nunca han recibido tratamiento (casos nuevos).

Resistencia secundaria o adquirida: pacientes con un tratamiento anterior con más de un mes de duración, ocasionada por el mal uso de los fármacos. Presenta resistencia a dos o más medicamentos y es frecuente la aparición de cepas con Multidrogoresistencia. (Farga, 2011)

\section{Conclusión}

La mayoría de los Pacientes con TBMDR corresponden a fracasos, recaídas, abandonos es necesario reforzar el seguimiento a los pacientes.

La resistencia es un fenómeno natural de defensa de los microorganismos, que si bien es controlable, es un fenómeno inevitable. 
Rev. SINAPSIS, Vol. 5, No 2, Diciembre 2014

ISSN $1390-7832$

El diagnóstico y tratamiento de los casos, ayuda en la cura del individuo, sin embargo este enfoque, no es suficiente para el control.

La generación de cepas con mayor perfil de resistencia, y su amplia extensión hacia la comunidad se está dando a nivel mundial, lo que incluye a nuestro país.

Necesitamos hacer esfuerzos intensos en garantizar el diagnóstico y tratamiento precoz, así como de la "adherencia total" a los diferentes esquemas de tratamiento TB.

\section{Bibliografía}

1. Fuentes-Tafur LA. Enfoque sociopolítico para el control de la tuberculosis en el Perú. Rev Peru Med Exp Salud Pública. 2009; 26(3): 370-79.

2. Monedero I. Caminero JA. Magnagement of multidrug-resistant tuberculosis: an udpate, ther adv Respir Dis. 2010; 4(2) 117-27.

3. ÁLVARO YÁNEZ DEL V. Tuberculosis en inmigrantes. Situación Chile-Perú. Rev Chil Enf Respir 2010; 26: 161-164. Médico integrante del Equipo Técnico de Tuberculosis, Dirección del Servicio de Salud Occidente. Ex Consultor Regional en Tuberculosis de la OMS/OPS.

4. Patricia, "Guía para la atención médica de pacientes con infección por VIH/SIDA en consulta externa y hospitales”, 4ed., 2009, pp: 76-82.*CENAVECE “Centro Nacional de Vigilancia Epidemiológica. (Sala de Juntas P.B)*Dra. Yvette Sánchez Vargas, Unidad de Medicina Familiar No 94, Medico Familiar.*Dr. Francisco Villegas, IMSS, Médico Familiar.*Centro Nacional para la Prevención y el Control del VIH/SIDA.

5. Hernández León C, Badia-Hernández F, Ponce de León A, Sierra MaderoJG, Martínez Gamboa A, Crabtree Ramírez B, Arredondo SB, GonzálezAguirre A, Guerrero Almeida ML, Bobadilla del Valle JM, González Rodríguez A, Sifuentes-Osornio J. Tuberculosis activa en una cohorte de reclusos infectados por VIH en una cárcel de la Ciudad de México: características clínicas y Epidemiológicas. Salud Pública de México [internet]. 2012; 54 [Consultado 2014feb 17].

6. Jassal M y Bishai W. Extensively drug-resistant tuberculosis. Lancet Infect Dis 2009; 9:1930.

7. http://www.salud.gob.mx/conasida. 